\title{
Epidemiologic Study of Primary Brain Tumors in Miyazaki Prefecture: A Regional 10-year Survey in Southern Japan
}

\author{
Fumitaka MATSUMOTO, ${ }^{1}$ Hideo TAKESHIMA, ${ }^{1}$ Shinji YAMASHITA, ${ }^{1}$ \\ Kiyotaka YOKOGAmi, ${ }^{1}$ Takashi WATANABE, ${ }^{1}$ Hajime OHTA, ${ }^{1}$ \\ and Miyazaki Brain Tumor Research Group \\ ${ }^{1}$ Department of Neurosurgery, Division of Clinical Neuroscience, Faculty of Medicine, \\ University of Miyazaki, Miyazaki, Japan
}

\begin{abstract}
The increased use of neuroimaging and the aging of society have changed the incidence and proportion of histological types of intracranial tumors in Japan. A population-based epidemiological survey has been reported only from Kumamoto Prefecture. We performed a 10-year survey in Miyazaki Prefecture to compare our findings with the incidence rate (IR) of primary intracranial tumors (PIT) reported in the Kumamoto survey. Our study included 1915 new cases of PIT diagnosed in Miyazaki Prefecture between 2007 and 2016. The crude IR was 16.97/100000/year. The most common tumor was meningioma $(46.3 \%)$, followed by glioma $(17.1 \%)$, pituitary adenoma (13.1\%), schwannoma (8.2\%), and malignant lymphoma (3.8\%). The age-specific IR of all PITs and of meningiomas, gliomas, pituitary adenomas, schwannomas, lymphomas, and germ cell tumors was similar in both prefectures. To directly compare with the age-adjusted IRs reported in the Kumamoto survey, we calculated the IR for the two prefectures. The age-adjusted IR of primary brain tumors in Miyazaki Prefecture was 14.65/100000/year, which was slightly higher than in the Kumamoto survey (14.09/100000/year between 1989 and 2008). The ageadjusted IR of glioma, schwannoma, and malignant lymphoma showed only a small difference between Miyazaki and Kumamoto. However, the age-adjusted IR of meningiomas was higher in Miyazaki than Kumamoto (6.15- vs. 4.97/100000/year), but the IR of pituitary adenoma was higher in Kumamoto than Miyazaki (2.66- vs. 2.13/100000/year). Although there were some differences between the two surveys, the IR of PIT showed a similar pattern in Kumamoto and Miyazaki, which are neighboring districts on Kyushu Island.
\end{abstract}

Keywords: intracranial tumor, epidemiological study, incidence

\section{Introduction}

The reported increase in the incidence of brain tumors is attributable to advances in diagnostic procedures using CT and MRI studies. These tumors tended to be observed in individuals with advanced age. ${ }^{1-4)}$ Short-term epidemiological studies have been conducted. ${ }^{5-8)}$ For example, a study from Nordic countries showed an increase in gliomas and

Received January 14, 2021; Accepted April 7, 2021

Copyright $\subseteq 2021$ The Japan Neurosurgical Society This work is licensed under a Creative Commons AttributionNonCommercial-NoDerivatives International License. meningiomas between 1974 and 2003 but revealed flattening trends in the years from 1998 to $2003{ }^{9)}$ As many societies are aging, the age-adjusted incidence rate (IR) of brain tumors must be investigated.

The Brain Tumor Registry of Japan, which was created to identify the statistical characteristics of brain tumors diagnosed since 1975, includes the number of brain tumors, their location, the rate of different types, and their survival rates but does not include the age-adjusted IR. The age-adjusted IR of primary brain tumors in Japan has been reported only for Kumamoto Prefecture where the overall age-adjusted IR increased from 9.47/100000/ year in $1989-1994^{10)}$ to $10.97 / 100000 /$ year in 1989 $1998^{4)}$ and to $14.09 / 100000 /$ year in $1989-2008 .^{11)}$ 
To determine whether regional surveys reflect the entire Japanese population, we conducted a 10-year (2007-2016) epidemiologic study in Miyazaki Prefecture and compared our findings with those of the Kumamoto survey.

\section{Patients and Methods}

\section{Study population}

The Institutional Review Board of Miyazaki University Hospital, Japan, approved our study (approval number 360). Miyazaki Prefecture (population of approximately 1100000) is located in southeastern Kyushu Island, which is the third largest Japanese island. It is adjacent to Kumamoto Prefecture (population of approximately 1860000); the land areas are similar in size (Miyazaki: $7735 \mathrm{~km}^{2}$, Kumamoto: $7409 \mathrm{~km}^{2}$ ). According to the decennial census, the annual population movement into or out of Miyazaki Prefecture between 2007 and 2016 was negligible (from $-0.97 \%$ to $+0.28 \%$ ).

In Miyazaki Prefecture, there are 16 hospitals or clinics associated with Miyazaki University Hospital. They have professional staff and modern medical facilities and are equipped to perform both diagnostic CT and MRI studies and to care for patients with intracranial tumors.

\section{Summary of registered patients}

Registration information on all new patients diagnosed at the 16 facilities between 2007 and 2016 included their age, sex, the district of their residence, the date of diagnosis, and the pathological diagnosis. All included information was carefully checked to avoid double registration. Residents were defined as individuals who resided within the physical limits of Miyazaki Prefecture for at least 1 year prior to diagnosis. For the incidence date, we used the first day of hospitalization during which the diagnosis was made for inpatients, or the date on which the CT or MRI studies resulted in a diagnosis of brain tumor for outpatients. Except for angiomas, we included all primary intracranial tumors (PITs) defined by the World Health Organization (WHO) classification of 2000.

\section{Incidence rate}

All data are based on the census data for Japan and Miyazaki Prefecture. Population figures were obtained from published decennial tabulations for Miyazaki Prefecture and Japan.

First, the crude annual IR was calculated from the total number of brain cancer patients and the total population of Miyazaki Prefecture and applied as the average IR over the 10 years (2007-2016) included in this study. The population of Miyazaki Prefecture for the 10-year study period ( $\mathrm{n}=1128411$ ) was taken as the average of the mid-year estimates for 2011 and 2012; population data were based on the 2011 and 2012 censuses.

To calculate the age-specific IR, we used the total number of patients diagnosed in each of the 10 years as the numerator and the population number for each age distribution, extracted from the Ministry of Internal Affairs and Communications data, as the denominator. The age-specific IR was calculated for all PITs and for each type of intracranial tumor.

The adjusted rates allow an overall comparison among different populations because the rates are standardized for dissimilarities in the age and sex distributions. To directly compare our findings with the reported age-adjusted IR in the Kumamoto survey for the years between 1989 and 2008, we calculated the age-adjusted IR for Miyazaki using the Japanese population estimate in the year 2000 as the standard, as was used in the Kumamoto survey. ${ }^{11)}$

\section{Results}

\section{Crude incidence}

During the 10-year period from 2007 to 2016, 1915 new cases of primary brain tumors were diagnosed and registered in Miyazaki Prefecture. Table 1 lists the type distribution of the 1915 intracranial tumors and the crude incidence of each tumor type. The most common tumors were meningiomas (46.3\%), followed by gliomas $(17.2 \%)$, pituitary adenomas (13.1\%), schwannomas (8.2\%), malignant lymphomas (3.8\%), and Rathke's cleft cysts $(2.9 \%)$. The crude IR for all PITs was 16.97/100000/year.

Of the 1915 intracranial tumors, $52.0 \%$ were confirmed histologically. Most tumors that were incidentally diagnosed clinically or radiologically were benign and tended to not be operated on at the time of their registration in the database. Therefore, the histological confirmation rate was 39.1\% for meningiomas, $46.8 \%$ for pituitary adenomas, and $27.3 \%$ for Rathke's cleft cysts (Table 1). The histological confirmation rate was higher for malignant tumors (gliomas: 81.3\%, malignant lymphomas: $66.7 \%$, germ cell tumors: $93.8 \%$, medulloblastomas, $100 \%$ ). Of the gliomas, $8 \%$ were asymptomatic, as was $1.4 \%$ of malignant lymphomas; no germ cell tumors and medulloblastomas were asymptomatic.

\section{Age-specific incidence rate}

The average annual sex- and age-specific incidence of all PITs is shown in Fig. 1. The IR of PITs increased in the first eight age groups (0-79 years); the patterns for males and females were similar. 
Table 1 Distribution of primary intracranial tumors in Miyazaki Prefecture between 2007 and 2016

\begin{tabular}{|c|c|c|c|c|c|c|}
\hline \multirow{2}{*}{ Tumor type } & \multicolumn{3}{|c|}{ Number of cases (\%) } & \multirow{2}{*}{$\begin{array}{c}\text { Crude } \\
\text { incidence/100000/ } \\
\text { year }\end{array}$} & \multirow{2}{*}{$\begin{array}{l}\text { Histological } \\
\text { confirmation } \\
\text { rate }(\%)\end{array}$} & \multirow{2}{*}{$\begin{array}{l}\text { Asymptomatic } \\
\text { ratio }(\%)\end{array}$} \\
\hline & Total & Male & Female & & & \\
\hline Meningioma & $886(46.3)$ & $218(30.3)$ & $668(55.9)$ & 7.86 & 39.1 & 66.9 \\
\hline Glioma & $329(17.2)$ & $180(24.8)$ & $149(12.5)$ & 2.89 & 81.3 & 8 \\
\hline Pituitary adenoma & $250(13.1)$ & $109(15.2)$ & $141(11.8)$ & 2.22 & 46.8 & 40.4 \\
\hline Schwannoma & $157(8.2)$ & $68(9.5)$ & $89(7.5)$ & 1.4 & 63.1 & 17.2 \\
\hline $\begin{array}{l}\text { Malignant } \\
\text { lymphoma }\end{array}$ & $72(3.8)$ & $43(6)$ & $29(2.4)$ & 0.64 & 66.7 & 1.4 \\
\hline Rathke's cyst & $55(2.9)$ & $18(2.5)$ & $37(3.1)$ & 0.49 & 27.3 & 59.3 \\
\hline Hemangioblastoma & $28(1.5)$ & $16(2.2)$ & $12(1)$ & 0.25 & 75 & 25 \\
\hline Craniopharyngioma & $25(1.3)$ & $10(1.4)$ & $15(1.3)$ & 0.22 & 64 & 20 \\
\hline Germ cell tumor & $16(0.8)$ & $14(1.9)$ & $2(0.2)$ & 0.14 & 93.8 & 0 \\
\hline Epidermoid & $15(0.8)$ & $8(1.1)$ & $7(0.6)$ & 0.13 & 60 & 46.2 \\
\hline Medulloblastoma & $11(0.6)$ & $4(0.5)$ & $7(0.6)$ & 0.1 & 100 & 0 \\
\hline Others & $71(3.7)$ & $33(4.6)$ & $38(3.1)$ & 0.63 & 33.8 & 54.9 \\
\hline Total & 1915 (100) & $721(100)$ & $1194(100)$ & 16.97 & 52 & 40.1 \\
\hline
\end{tabular}

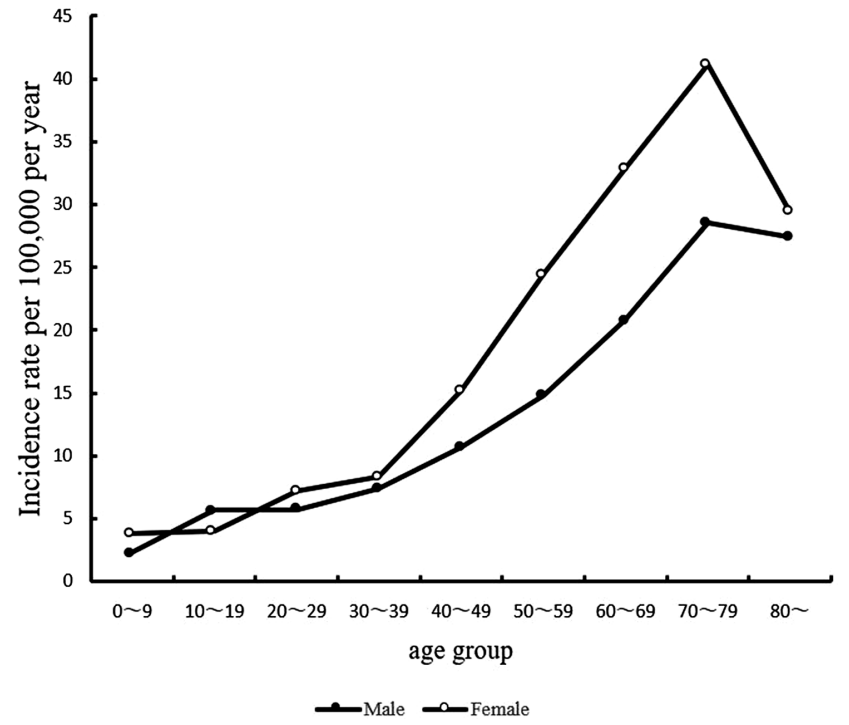

Fig. 1 Graph shows the average annual sex- and age-specific incidence of intracranial tumors in Miyazaki Prefecture. Closed circle: male, open circle: female.

Males and females in the 70-79-year group manifested the highest IR; the IR was 28.51/100000/year for men and 41.17/100000/year for women. In all age groups except for individuals aged 10-19 years, the IR was slightly higher in females than males.

As shown in Fig. 2A, the IR of meningiomas increased with age in both males and females. However, in the 20-29-year-old group, it was slightly higher in males than females (males, 0.82/100000/ year; females, 0.36/100000/year). With advancing age, the meningioma IR showed a marked gender difference. Among individuals aged 70-79 years, the peak IR was 28.44/100000/year in females vs. $12.93 / 100000 /$ year in males.

The age-specific IR of gliomas peaked at 5.67/100000/ year in males in the 70-79-year group and at $4.34 / 100000 / y e a r$ in females older than 80 years (Fig. 2B). In most age groups, the IR of glioma was higher in males than females.

In males, the rate of pituitary adenomas increased with age; it peaked at 3.71/100000/year in 70 - to 79-year-old males. In females, we noted three peaks. The first was 2.55/100000/year in 30-39-year-old women; the second was 4.19/100000/year (50-59 years of age); and the third peak was 3.49/100000/ year in women aged 70-79 years (Fig. 2C) and lower than in males of the same age.

In our survey, $79 \%$ of schwannomas originated at a vestibular nerve; $63.1 \%$ of these tumors were histologically confirmed. The age-specific peak IR was 2.66/100000/year in males aged 70-79 years; it was 3.30/100000/year in females 60-69 years of age (Fig. 2D).

The incidence of malignant lymphomas increased with age, especially in males. In males and females older than 60 years, the incidence was markedly increased. The peak incidence was 2.53/100000/ year in males older than 70 years; in females between the age of 70 and 79 years, it was 1.23/100000/year (Fig. 2E). 

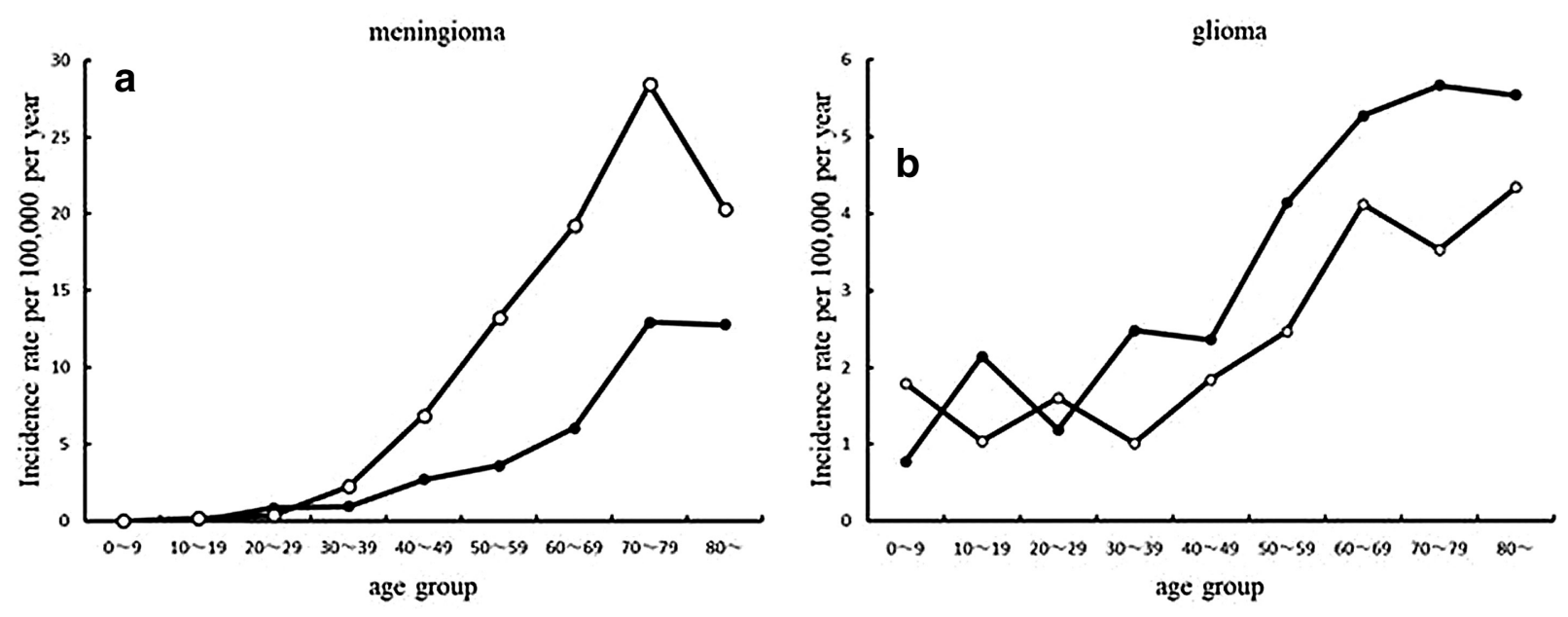

$\rightarrow$ Male $\infty$ Fermale

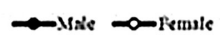

pituitary adenoma
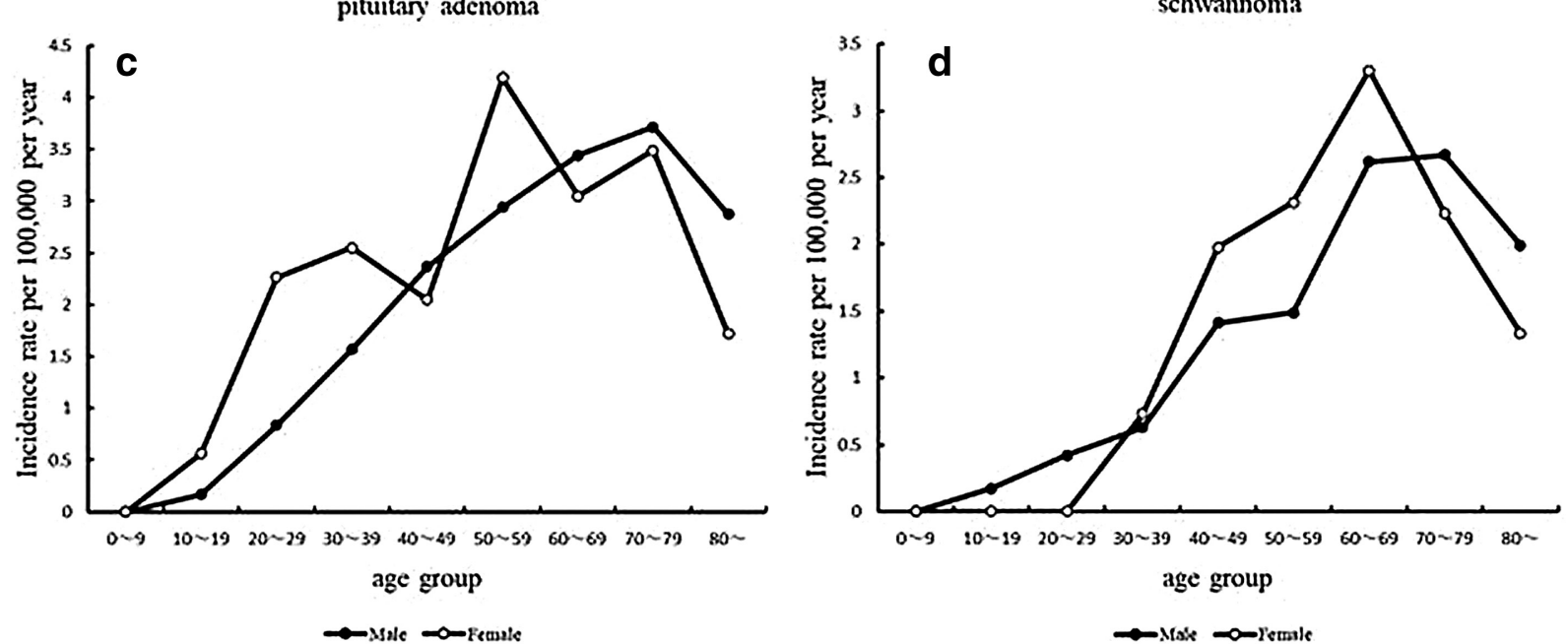

malignant lymphoma
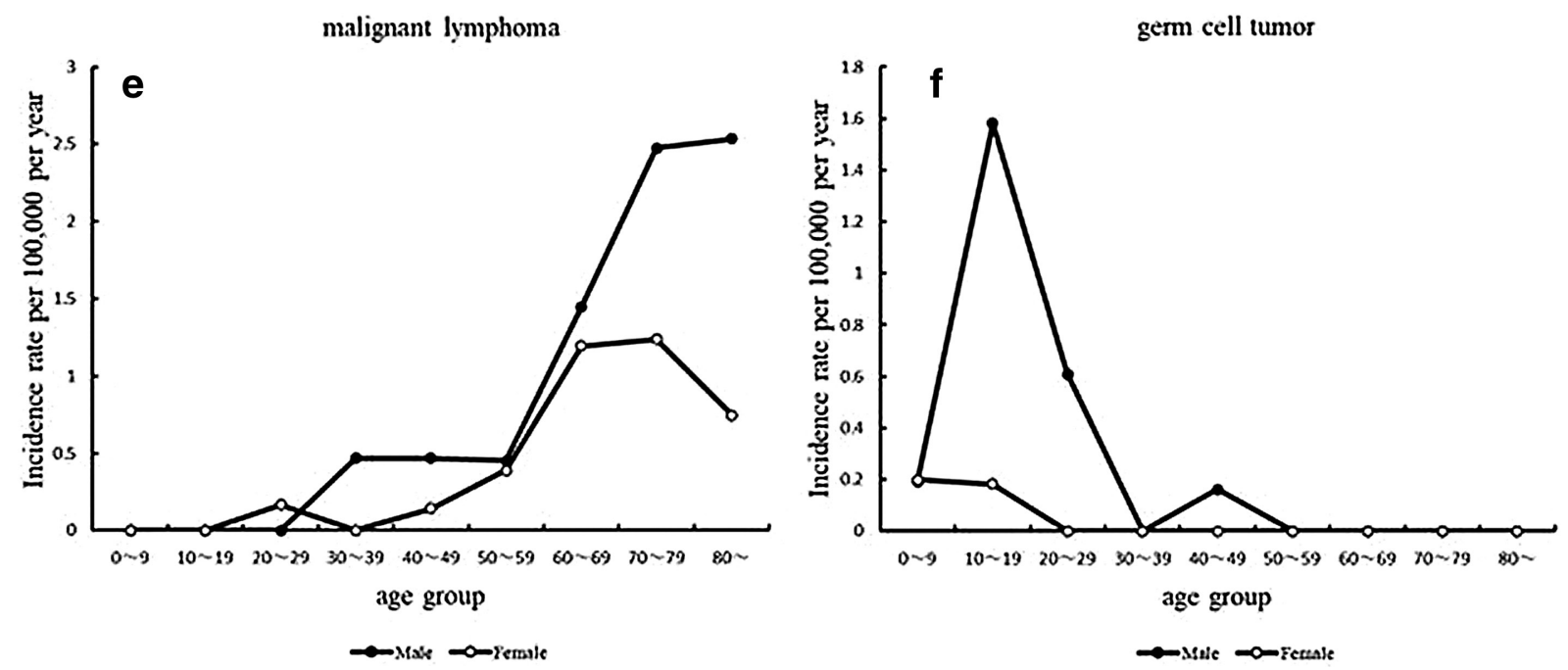

Fig. 2 Graph shows the average annual sex- and age-specific incidence rates in Miyazaki Prefecture. (a) Meningioma, (b) glioma, (c) pituitary adenoma, (d) schwannoma, (e) malignant lymphoma, (f) germ cell tumor. 
The peak incidence of germ cell tumors was $1.58 / 100000 /$ year in males aged 10-19 years. It was 8.6 times higher than in females of the same age (Fig. 2F).

\section{Age-adjusted IR}

As shown in Table 2, the average annual age-adjusted IR of PITs diagnosed between 2007 and 2016 was $14.65 / 100000 / y e a r$, which was slightly higher than in the Kumamoto survey (14.09/100000/year [1989-2008]). Among males residing in Miyazaki Prefecture, the age-adjusted incidence was 12.05/100000/year; among females, it was 16.89/100000/year.

Among PITs, meningiomas had the highest IR. The age-adjusted IR was 6.15/100000/year (males: 3.36-, females: 8.56/100000/year). The next highest IR was for gliomas; the age-adjusted IR was 2.59/100000/year (males: 3.03-, females: 2.19/100000/year). Males had a higher IR of gliomas, malignant lymphomas, hemangioblastomas, germ cell tumors, and epidermoid cysts. Females had a higher IR of meningiomas, pituitary adenomas, schwannomas, Rathke's cleft cysts, craniopharyngiomas, and medulloblastomas. The IR of meningiomas was 2.5 times higher in females than in males. In comparison, the IR of germ cell tumors was 7.5 times higher in males than in females.

\section{Discussion}

This is the first population-based study of primary brain tumors from Miyazaki Prefecture. During the

Table 2 Age-adjusted incidence rate using the year 2000 Japanese population as a standard

\begin{tabular}{lcccc}
\hline \multirow{2}{*}{\multicolumn{1}{c}{ Tumor type }} & \multicolumn{2}{c}{ Incidence/100000/year } & M/F \\
\cline { 2 - 4 } & Total & Male & Female & \\
\hline Meningioma & 6.15 & 3.36 & 8.56 & 0.39 \\
Glioma & 2.59 & 3.03 & 2.19 & 1.38 \\
Pituitary adenoma & 2.13 & 1.91 & 2.33 & 0.82 \\
Schwannoma & 1.24 & 1.17 & 1.32 & 0.88 \\
Malignant & 0.49 & 0.66 & 0.37 & 1.78 \\
lymphoma & & & & \\
Rathke's cyst & 0.54 & 0.34 & 0.72 & 0.48 \\
Hemangioblastoma & 0.26 & 0.3 & 0.21 & 1.43 \\
Craniopharyngioma & 0.22 & 0.18 & 0.26 & 0.67 \\
Germ cell tumor & 0.17 & 0.3 & 0.04 & 7.74 \\
Epidermoid & 0.13 & 0.15 & 0.12 & 1.28 \\
Medulloblastoma & 0.11 & 0.08 & 0.15 & 0.51 \\
Others & 0.62 & 0.61 & 0.6 & 1.02 \\
All tumor & 14.65 & 12.05 & 16.89 & 0.71 \\
\hline
\end{tabular}

10-year period from 2007 to 2016, 1915 cases of primary brain tumors were newly diagnosed in Miyazaki Prefecture. The crude IR for all PITs was 16.97/100000/year.

Currently, the only reliable regional Japanese survey is available from Kumamoto Prefecture. As Miyazaki and Kumamoto prefectures are adjacent areas on Kyushu Island and although Miyazaki is more rural than Kumamoto and its aged population is greater than that of Kumamoto, we performed a 10-year epidemiologic survey in Miyazaki Prefecture to compare the IR of PITs in the two prefectures.

To directly compare our findings with the reported age-adjusted IR for Kumamoto, we calculated the age-adjusted IR using the Japanese population estimate from the year 2000 as the standard, as was used in the Kumamoto study, rather than the mid-year census data. ${ }^{11)}$ We found that the average annual age-adjusted IR of PITs was 14.65/100000/year in Miyazaki, which was considerably lower than the crude IR (16.97/100000/year). This observation can be explained by the progressive aging of Japanese society and by population movement from provinces to big cities over time.

The age-specific IR of primary brain tumors in both prefectures exhibited a similar pattern for not only all PITs but also for meningiomas, gliomas, pituitary adenomas, schwannomas, lymphomas, and germ cell tumors. However, the age-adjusted IR of primary brain tumors was slightly higher in Miyazaki than Kumamoto (14.65/100000/year between 2007 and 2016 vs. 14.09/100000/year between 1989 and 2008). This small difference may be attributable to the different periods during which the surveys were conducted. Three reports in the Kumamoto survey showed that the age-adjusted IR gradually increased. In the report covering the years from 1989 to 1994, the IR was 9.47/100000/year, ${ }^{10)}$ and between 1989 and 1998 , it increased to $10.97 / 100000 /$ year. In the period from 1989 to 2008, it was even higher (14.09/100000/year).,11) The close age-adjusted IRs suggest that both surveys yielded reliable data.

Although there was only a small inter-prefecture difference in the rate of gliomas, schwannomas, and malignant lymphomas, there was a marked difference in the rate of meningiomas (Miyazaki: 46.3\%, Kumamoto: 36.8\%). In Miyazaki, 886 of 1915 PITs (46.3\%) were meningiomas; 668 of 1194 (55.9\%) brain tumors diagnosed in women, who tend to live longer than men, were meningiomas.

For a direct inter-prefecture comparison, we calculated the age-adjusted IR using the Japanese population estimate from 2000 as the standard. Even after compensation, the age-adjusted IR of meningiomas in Miyazaki was higher than in Kumamoto 
(6.15- vs. $4.97 / 100000 / y e a r)$. It is possible that the number of CT and MRI studies of asymptomatic patients was higher in Miyazaki than Kumamoto institutions; histological meningioma confirmation was obtained in $39.1 \%$ of Miyazaki and $47 \%$ of Kumamoto patients.

According to published epidemiological surveys conducted in several countries, the age-adjusted IR of primary brain tumors ranges from 8.7 to 18.1/100000/year. ${ }^{8,10,12)}$ Calculating the age-adjusted IR using a common standard population allows for comparisons of rates across regions with different age structures. Central Brain tumor Registry of the United States (CBTRUS) reported that the age-adjusted IR of total primary brain (malignant and non-malignant) tumors was 18.16/100000/year, which is adjusted to the U.S. standard population in 2000. Our calculation of age-adjusted IR of PITs was $14.65 / 100000 / y e a r$, which is slightly lower in Miyazaki than in the U.S.

In comparing the age-adjusted IR for tumor types between the U.S. and our data, we found that the IRs of meningiomas (6.03/100000/year in U.S. vs. 6.15/100000/year in Miyazaki), pituitary adenomas $(2.22 / 100000 /$ year in U.S. vs. $2.13 / 100000 /$ year in Miyazaki), and schwannomas (1.57/100000/year in U.S. vs. 1.24/100000/year in Miyazaki) were similar. However, there was a difference in IRs for gliomas (3.18/100000/year glioblastomas only in U.S. vs. $2.59 / 100000 /$ year gliomas total in Miyazaki) and germ cell tumors $(0.08 / 100000 /$ year in U.S. vs. $0.17 / 100000 /$ year in Miyazaki). ${ }^{12)}$

In addition, the calculated age-adjusted IR of glioblastomas for Miyazaki was 1.26/100000/year. The most recent literature from the U.S. reported age-adjusted IRs by race categories including White, Black, American Indian/Alaskan Native (AIAN), and Asian/Pacific Islander (API). The rate of glioblastomas was highest in White (3.49/100000/year) compared to Black (1.79/100000/year), AIAN (1.43/100000/year), and API (1.46/100000/year) populations. ${ }^{13)}$ Interestingly, the age-adjusted IRs of people of color in our research was almost similar.

Other studies have reported that the incidence of germ cell tumors is higher in East Asia, including Japan, than in Western countries. ${ }^{8,12,14)}$ The incidence of central nervous system (CNS) germ cell tumors in the year 2000 was $0.023 / 100000 /$ year in the country of Georgia. ${ }^{8)}$ From 1989 to 1990, its crude incidence was 0.1/100000/year in Scottish Lothian. ${ }^{2}$ McCarthy et al. found that the incidence of primary CNS germ cell tumors in the Japan Cancer Surveillance Research Group (JCSRG) and the U.S. was not significantly different. ${ }^{15)}$ However, our findings suggest that the IR of germ cell tumors was somewhat higher in Japan than in Western countries. According to our Miyazaki survey, the age-adjusted IR of germ cell tumors was $0.17 / 100000 /$ year. In Kumamoto, the age-adjusted IR between 1989 and 1994 was $0.17 / 100000 /$ year; it was $0.18 / 100000 /$ year between 1989 and 2008. There was no significant inter-prefecture difference. The JCSRG reported that the incidence of CNS germ cell tumors decreased over time in males; in females, it remained the same. The IR was 0.096/100000/year (males: 0.143-, females: 0.046/100000/year). However, their database analysis is not representative of the entire Japanese population.

Our study has some limitations. It is a relatively small regional study (population of approximately 1100000), and only $52 \%$ of the tumors were histologically confirmed. Many non-malignant brain tumors were not confirmed by histology; the confirmation rate for meningiomas was $39.1 \%$. However, advances in neuroimaging rendered a definitive diagnosis of meningioma by CT or MRI possible, and the wide availability of neuroimaging instrumentation facilitates the non-invasive tracking of tumor growth. With the aid of CT and MRI, our knowledge of the natural history of meningiomas will expand. ${ }^{16)}$

\section{Conclusion}

We surveyed 1915 new cases of PIT diagnosed in Miyazaki Prefecture between 2007 and 2016. The age-adjusted IR of primary brain tumors was $14.65 / 100000 / y e a r$, which was similar to the finding in the Kumamoto survey (14.09/100000/year between 1989 and 2008). Although the inter-prefecture difference was small, the age-adjusted IR of meningiomas was higher in Miyazaki than Kumamoto (6.15- vs. $4.97 / 100000 /$ year). In contrast, the incidence of pituitary adenomas was lower in Miyazaki than Kumamoto (2.13- vs. 2.66/100000/year).

\section{Acknowledgments}

The following hospitals or clinics in Miyazaki Prefecture contributed to this epidemiological study: Bethesda Clinic, Ikeda Hospital Neurosurgical Service, Junwakai Memorial Hospital, Kanemaru Neurosurgery Hospital, Miyakonojo Medical Association Hospital, Miyazaki Prefectural Miyazaki Hospital, Miyazaki Prefectural Nichinan Hospital, Miyazaki Prefectural Nobeoka Hospital, Noguchi Neurosurgical Hospital, Nonaka Clinic, Ohyama Neurosurgical Clinic, Saito Koyu Medical Center, Ueda Neurosurgical Hospital, Wada Hospital, and Yoneda Neurosurgical Clinic. We thank Ursula Petralia for editing assistance. 


\section{Conflicts of Interest Disclosure}

The authors report that they have no conflicts of interest. All the authors who are members of The Japan Neurosurgical Society (JNS) filed online self-reported COI Disclosure Statement Forms online via the JNS member website.

\section{References}

1) Mao $Y$, Desmeules M, Semenciw RM, Hill G, Gaudette L, Wigle DT: Increasing brain cancer rates in Canada. Can Med Assoc J 145: 1583-1591, 1991

2) Counsell CE, Collie DA, Grant R: Incidence of intracranial tumours in the Lothian region of Scotland, 1989-90. J Neurol Neurosurg Psychiatry 61: 143-150, 1996

3) Lönn S, Klaeboe L, Hall P, et al.: Incidence trends of adult primary intracerebral tumors in four Nordic countries. Int J Cancer 108: 450-455, 2004

4) Kuratsu J, Takeshima H, Ushio Y: Trends in the incidence of primary intracranial tumors in Kumamoto. Japan Int J Clin Oncol 6: 183-191, 2001

5) Ahmad OB, Boschi-Pinto C, Lopez AD, Murray CJL, Lozano R, Inoue M: Age standardization of rates: a new WHO standard. GPE discussion paper series: World Health Organization No. 31, 2001

6) Dolecek TA, Dressler EVM, Thakkar JP, Liu M, Al-Qaisi A, Villano JL: Epidemiology of meningiomas post-Public Law 107-206: The Benign Brain Tumor Cancer Registries Act. Cancer 121: 2400-2410, 2015

7) Dobec-Meić B, Pikija S, Cvetko D, et al.: Intracranial tumors in adult population of the Varazdin County (Croatia) 1996-2004: a population-based retrospective incidence study. J Neurooncol 78: 303-310, 2006

8) Gigineishvili D, Shengelia N, Shalashvili G, Rohrmann S, Tsiskaridze A, Shakarishvili R: Primary brain tumour epidemiology in Georgia: first-year results of a population-based study. J Neurooncol 112: 241-246, 2013

9) Deltour I, Johansen C, Auvinen A, Feychting M, Klaeboe L, Schüz J: Time trends in brain tumor incidence rates in Denmark, Finland, Norway, and Sweden, 1974-2003. J Natl Cancer Inst 101: 1721-1724, 2009

10) Kuratsu J, Ushio Y: Epidemiological study of primary intracranial tumors: a regional survey in Kumamoto Prefecture in the southern part of Japan. J Neurosurg 84: 946-950, 1996

11) Nakamura H, Makino K, Yano S, Kuratsu J: Kumamoto Brain Tumor Research Group: Epidemiological study of primary intracranial tumors: a regional survey in Kumamoto prefecture in southern Japan--20-year study. Int J Clin Oncol 16: 314-321, 2011

12) Central Brain Tumor Registry of United states (CBTRUS) Statistical Report: Primary brain and central nervous system tumors diagnosed in the United States in 2004-2005

13) Ostrom QT, Cioffi G, Gittleman H, et al.: CBTRUS statistical report: Primary brain and other central nervous system tumors diagnosed in the United States in 20122016. Neuro Oncol 21(Suppl 5): v1-v100, 2019

14) Pobereskin LH, Chadduck JB: Incidence of brain tumours in two English counties: a population based study. J Neurol Neurosurg Psychiatry 69: 464-471, 2000

15) McCarthy BJ, Shibui S, Kayama T, et al.: Primary CNS germ cell tumors in Japan and the United States: an analysis of 4 tumor registries. Neuro Oncol 14: 11941200, 2012

16) Huang RY, Bi WL, Griffith B, et al.: Imaging and diagnostic advances for intracranial meningiomas. Neuro Oncol 21 (Suppl 1): i44-i61, 2019

Corresponding author: Fumitaka Matsumoto, MD Department of Neurosurgery, Division of Clinical Neuroscience, Faculty of Medicine, University of Miyazaki, 5200 Kiyotakechokihara, Miyazaki 889-1692, Japan. e-mail: fumitaka_matsumoto@med.miyazaki-u.ac.jp 